COMMENT. Congenital fiber type disproportion (CFTD) is a rare cause of congenital myopathy and hypotonia. Clinical features are heterogeneous, and mutations in several genes have been identified. Diagnosis should exclude other causes for myopathy, since type 1 fiber hypotrophy is a common secondary feature in many neuromuscular disorders. The above authors have previously found mutations in ACTA1 and SEPN1 genes in a few patients with CFTD. The present report of TPM3 mutations involving 11 cases in 5 families is the largest series to date. Affected patients present with hypotonia before the first birthdate and show a slow progression of proximal muscle weakness, kyphoscoliosis, and respiratory insufficiency, but most remain ambulant and survive to adulthood.

\title{
DIAGNOSTIC APPROACH TO NEONATAL HYPOTONIA
}

The frequency of various disorders causing neonatal hypotonia and the reliability of the first physical examination and standard diagnostic tests were evaluated by a retrospective review of records of 144 patients diagnosed between 1999 and 2005 at Strasbourg University Hospital, France. Of 120 cases with a final diagnosis of neonatal hypotonia, $82 \%$ had central (cerebral) causes, including hypoxic and hemorrhagic brain lesions in $34 \%$, chromosomal abnormalities (eg Prader-Willi syndrome) in $26 \%$, brain malformations in $12 \%$, and metabolic or endocrine diseases in $9 \%$. Peripheral (neuromuscular) causes confirmed in 22 $(18 \%)$ cases included spinal muscular atrophy in $6 \%$ and myotonic dystrophy in $4 \%$. Hypotonia first noticed before the $28^{\text {th }}$ day of life and lasting for at least two weeks was an inclusion criterion. Exclusion criteria were gestational age less than 35 weeks, neonatal infection and congenital heart disease. Initial presentation of hypotonia was classified as central, peripheral or undetermined, according to Dubowitz criteria. Central hypotonic cases had preserved antigravity limb movements, normal or increased peripheral tone, poor visual contact, seizures, and brisk tendon reflexes. Peripheral hypotonia was characterized by muscular weakness, absent antigravity movements, decreased reflexes, global hypotonia, and preserved social interaction. Hypotonia was diagnosed in $4.2 \%$ of neonates admitted. Mean age of referral was 11.8 days. Swallowing difficulties affected $101(70 \%)$, and respiratory distress occurred in $79(55 \%)$.

The initial neurologic examination classified hypotonia as central in $87(60 \%)$, peripheral in $40(28 \%)$, and undetermined in 17 cases. The positive predictive value of the first clinical examination was $86 \%$ for central hypotonia and $52 \%$ for peripherai cases. Among 17 with undetermined initial diagnosis, 14 cases proved to be of central origin. Decreased fetal movements and/or polyhydramnios were reported in 19 pregnancies $(13 \%)$, and were predictive of a prenatal cause in $15(\mathrm{p}<0.05)$. Perinatal asphyxia in 77 cases was the cause in 39 cases. At time of follow-up (1 year or longer) $40(29 \%)$ infants had died (22 in the first two months), and $8(6 \%)$ were completely recovered. Risk factors for a higher mortality rate were initial respiratory distress, prolonged feeding difficulties, and neuromuscular causes for hypotonia $(\mathrm{p}<0.05)$. Neuroimaging contributed to the final diagnosis in 50 cases, especially for brain malformation (MRI), intracranial hemorrhage (CT), and hypoxic-ischemic encephalopathy. EEG contributed to diagnosis in $35 / 92$ (38\%) cases, especially with hypoxic and/or hemorrhagic brain lesions and cortical gyration abnormalities. DNA-based diagnostic tests performed in 43 cases were confirmatory in 18 $(42 \%)$. Molecular tests in 34 cases confirmed a diagnosis of spinal muscular atrophy in 5 , myotonic dystrophy in 5, and Prader-Willi syndrome in 3 cases. Karyotype analyses in 59 
neonates were contributory in $24(41 \%)$. Metabolic tests in 45 cases contributed to the final diagnosis in $9(20 \%)$. EMG and NCS in 23 neonates were contributory in 10, but misleading and incorrect in 3 (spinal muscular atrophy mistaken for demyelinating neuropathy, and negative reports in a case of myotonic dystrophy and one of congenital muscular dystrophy). Muscle biopsy in 14 cases was helpful in 6, and tests for congenital myasthenia in 10 were negative. (Laugel V, Cossee $\mathrm{M}$, Matis $\mathbf{J}$ et al. Diagnostic approach to neonatal hypotonia: retrospective study on 144 neonates. Eur J Pediatr May 2008;167:517-523). (Respond: Dr Vincent Laugel, Sevice de Pediatrie 1, CHU Strasbourg-Hautepierre, Avenue Moliere, F67098 Strasbourg Cedex, France. E-mail: vincent.laugel(a) chru-strasbourg.fr).

COMMENT. The hypotonic infant is a fairly common pediatric problem, accounting for 1 in 25 admissions to a tertiary medical center and neonatal unit. The authors provide a diagnostic algorithm based on the yield of the initial neurological examination and various tests. Central (cerebral) causes are most frequent, and the initial physical examination has a higher positive predictive value for this type of hypotonia than for peripheral (neuromuscular) types. First line tests to confirm a central cause are neuroimaging and EEG. For peripheral type hypotonia, examination of the mother (in suspected myotonic dystrophy or neonatal transient myasthenia) and DNA-based tests are most reliable. In some cases a longer follow-up may be necessary to determine the course of the disease and the correct diagnosis.

The hypotonic or limp infant syndrome has been reviewed in the literature over many years. (Walton JN. The limp child. J Neurol Neurosurg \& Psychiat 1957;20:144-154) (Millichap JG. The hypotonic child. In Practice of Pediatrics. Vol IV, ed. BrennemannKelley, Chap 16, Hagerstown, MD; WF Prior Co. 1966). Infants with so-called benign congenital hypotonia, a term coined by Walton, are limp at birth, sitting and walking are delayed, but muscle wasting is not profound. The muscle fibers are generally small for the age. Muscle strength and motor development gradually improve, but mental retardation and other congenital abnormalities are not uncommon. In recent years, molecular genetic diagnosis of myopathies has uncovered several inherited diseases characterized by neonatal hypotonia, including nemaline myopathy and congenital fiber type disproportion (Clarke NF et al. Ann Neurol March 2008;63:329-337) (Rifai Z et al. Neurology 1993;43:2372-2377). Defining the genetic basis of these diseases will explain their heterogeneous clinical manifestations anu lead to improvements in family counseling.

\section{MOVEMENT DISORDERS}

\section{DOPA-RESPONSIVE DYSTONIA WITH DELAY IN WALKING}

A 2-year, 8-month old boy with a previous diagnosis of cerebral palsy was referred to UCSF because of "awkward" gait and "walking on the toes." His gait abnormality progressed, worsening in the evening, and he was found to have a dopa-responsive dystonia caused by an autosomal-dominant $\mathrm{GCH} 1$ mutation. He was treated successfully with oral carbadopa-levodopa (Sinemet). Three other family members were affected, presenting with

stiffness in the thighs, motor impairment, speech and swallowing difficulties, postural tremor and depressive anxiety, also responsive to carbadopa-levodopa. (Cheyette BNR et al. Pediatr Neurol April 2008;38:273-275). 